\title{
Enhanced Position Estimation via Node Cooperation
}

\author{
Zafer Sahinoglu*, Sinan Gezici* \\ $\star$ Mitsubishi Electric Research Laboratories, 201 Broadway Avenue, Cambridge, MA 02139, USA \\ * Dept. of Electrical and Electronics Engineering, Bilkent University, Bilkent, Ankara 06800, Turkey \\ Emails: zafer@merl.com, gezici@ee.bilkent.edu.tr
}

\begin{abstract}
Two-way time-of-arrival (TW-ToA) is a widely used ranging protocol that can provide the distance between two devices without time synchronization. One drawback of the TWToA is poor positioning accuracy in the absence of a sufficient number of reference ranging devices. Also, for a self-positioning system with a limited battery life, it might be necessary to limit the number of transmissions while satisfying accuracy constraints. In this paper, a cooperative positioning protocol [1] is studied, which can improve positioning accuracy compared to the conventional TW-ToA based positioning systems and also facilitate positioning with fewer packet transmissions; hence, it can prolong battery life on average. The maximum likelihood estimator is obtained for the cooperative technique and the limits on the positioning accuracy are quantified in terms of the Cramer-Rao lower bound (CRLB). Simulation results are provided in order to show performance improvements.

Index Terms- Cooperative positioning, time-of-arrival (ToA), maximum likelihood, Cramer-Rao lower bound (CRLB).
\end{abstract}

\section{INTRODUCTION}

An increasing number of applications benefit from location awareness [2]-[5]. While the Global Positioning System (GPS) offers positioning accuracy on the order of several meters, it is not a viable solution for indoor scenarios due to blocked satellite signals leading to severe non-line-of-sight (NLoS) errors and long acquisition times [2]. Various real-time location systems (RTLSs) are commercially available for indoor applications [6], [7]. These systems adopt time-difference-of-arrival (TDoA) based positioning scheme, and consist of transmitonly tags and time synchronized anchors. Synchronization can be handled either via transmitting periodic beacons wirelessly from a designated device so-called the coordinator, or wiring all the reference devices (or, anchors) and feeding them with a common clock [6]-[8].

The two-way time-of-arrival (TW-ToA) based positioning system offers better positioning accuracy than the TDoA, given the same number of reference devices [9]. In addition, the TW-ToA does not have the stringent time synchronization requirement. However, it generates more air traffic (i.e., employs more packets) and increases overall energy consumption of the RTLS network. Traffic volume and energy inefficiencies of the TW-ToA need to be lowered in order to make TW-ToA based systems commercially attractive. To that aim, a cooperative positioning algorithm is proposed in [1], which involves cooperation between reference nodes (RNs) in order to reduce the signaling overhead, but optimal position estimators and theoretical performance limits have not been studied.

For most of the cooperative localization schemes in the literature, the particular emphasis is to increase the accuracy of

${ }^{0} \mathrm{~S}$. Gezici wishes to acknowledge the activity of the Network of Excellence in Wireless COMmunications NEWCOM++ of the European Commission (contract n. 216715) that motivated this work. initial position estimates either via information sharing among nodes that are being located [10], [11] or data fusion of multiple received signal strength (RSS), angle-of-arrival (AoA) and ToA measurements at multiple nodes [12], [13]. In [14], a cooperative non-parametric belief propagation algorithm is developed to determine the infrastructure map. Even though the approach suffers from mobility, it complements the study in [1], which assumes that the locations of the anchor nodes are known a-priori. Another complementary work [15] introduces a distributed algorithm to mitigate propagation of anchor location estimation errors. It is possible to use the position estimates obtained by means of cooperative algorithms in initializing the algorithms in [14] and [15].

In this paper, the cooperative positioning algorithm in [1] is studied from a theoretical perspective. First, a mathematical model is obtained for the measurements that are obtained at various RNs, and the statistics of measurement errors are specified. Then, the optimal maximum likelihood estimator is obtained for position estimation based on ToA and time difference measurements at different RNs. In addition, the Cramer-Rao lower bound (CRLB) is derived in order to quantify the performance limits. It is also shown via simulations that the cooperative positioning algorithm improves positioning accuracy with respect to the conventional TWToA, and is capable of estimating target positions with fewer numbers of transmissions than the TW-ToA. The reduction in the number of transmissions is useful especially for selfpositioning (navigation) systems under power constraints [9].

The remainder of the paper is organized as follows: Section II describes time-of-flight (equivalently, distance) estimation in the conventional TW-ToA ranging protocol. Section III provides a detailed description of the cooperative positioning algorithm and provides closed form expressions for both the maximum likelihood position estimator and performance bounds. In Section IV, simulation results are presented, followed by concluding remarks in Section V.

\section{TW-TOA RANGING PROTOCOL}

In this study, we adopt the terminology used in the IEEE 802.15.4a standard [16], and refer to a ranging capable device and the signals used for ranging as RDEV and RFRAME, respectively. The range between two $R D E V \mathrm{~s}$ can be determined simply via two-way exchanging of RFRAMEs and tracking their arrival times. This particular method is called the twoway time-of-arrival (TW-ToA) ranging protocol [17].

Assume that $R D E V A$ wants to perform ranging with $R D E V$ $B$, and that they remain stationary. The true elapsed time, $T_{r}$, between the departure of the outgoing RFRAME from $A$ and the arrival of the reply $R F R A M E$ from $B$ is given by $T_{r}=$ 
$2 T_{f}+T_{t a}^{B}$, where $T_{f}$ is the one-way time-of-flight (ToF) of the signal, and $T_{t a}^{B}$ is the turn-around time introduced at $B$.

It is possible to measure the time instant that the outgoing frame leaves the transmit antenna via loop-back tests and time calibrations. Therefore, transmit time-stamps can be assumed to be very close to their true values. Thus, in practice, any error in detecting the ToA of an incoming frame determines the accuracy of the estimate of the turn-around time, $\hat{T}_{t a}^{B}$. Timing imperfections even in the order of nanoseconds, multipath fading and NLoS propagation can easily induce undesirably large positive bias in a range estimate $(30 \mathrm{~cm}$ per nanosecond). In [18], the ToA based range estimation error $n_{i}$ is modeled as a zero mean Gaussian random variable with variance $\sigma_{i}^{2}$; that is $n_{i} \sim \mathcal{N}\left(0, \sigma_{i}^{2}\right)$, where $\mathcal{N}\left(x_{1}, x_{2}\right)$ represents a Gaussian random variable with mean $x_{1}$ and variance $x_{2}$. ToA estimation errors at two different devices are independent. The round-trip-time $\hat{T}_{r}$, measured at $A$, is then given by $\hat{T}_{r}=2 T_{f}+T_{t a}^{B}+n_{A}$, where the random variable $n_{A}$ is added due to ToA estimation at $A$.

In practice, $B$ obtains an estimate of the turn-around time, $\hat{T}_{t a}^{B}$, by calculating the difference between the estimated arrival time of the incoming frame and recorded departure time of the outgoing frame. Then, $B$ reports $\hat{T}_{t a}^{B}$ to $A$. Note that the estimation of $\hat{T}_{t a}^{B}$ at $B$ involves the ToA estimation of the incoming frame and the measurement of the time instant that the outgoing frame leaves the transmit antenna. As discussed above, ToA estimation is accompanied with a zero mean Gaussian error, namely, $n_{B} \sim \mathcal{N}\left(0, \sigma_{B}^{2}\right)$, whereas the recorded departure time can be considered as the true value since it can be accurately determined via loop-back tests and time calibrations. Therefore, the estimate of the turn-around time is modeled as $\hat{T}_{t a}^{B} \sim \mathcal{N}\left(T_{t a}^{B}, \sigma_{B}^{2}\right)$. Finally, $A$ estimates the one-way ToF as $\hat{T}_{f}=\left(\hat{T}_{r}-\hat{T}_{t a}^{B}\right) / 2$. Then, it can be obtained that $\hat{T}_{f} \sim \mathcal{N}\left(T_{f}, \frac{\sigma_{A}^{2}+\sigma_{B}^{2}}{4}\right)$.

\section{The Cooperative Positioning Protocol}

The TW-ToA and TDoA based techniques are two common ranging protocols to determine target positions in RTLSs [4]. TDoA based RTLSs require an infrastructure of synchronized readers. The target to be located transmits a beacon, and each reader that receives the beacon records its arrival time. As all the receivers are driven by a common clock, the difference of the arrival times at any two readers specifies a hyperbola. Similarly, in ToA based RTLSs, each TW-ToA measurement with a reader specifies a circle. Intersection of multiple circles in the TW-ToA or, of multiple hyperbolas in the TDoA is searched, and claimed as the target position [9]. With four readers and one target, the TDoA RTLS generates one wireless transmission, whereas for the TW-ToA RTLS it is eight. This provides some insight into the traffic inefficiency of the latter. In dense networks with multiple targets, the TWToA may become inoperable due to collisions. On the other hand, the main disadvantage of the TDoA based scheme is the need and cost for synchronization and calibration among reference devices. In this section, a cooperative positioning protocol is studied, which does not require synchronization among reference devices, but offers improved accuracy and/or reduced complexity compared to the conventional TW-ToA based scheme [1].
In the cooperative positioning system, three types of devices are defined: Primary reference node $(\mathrm{RN})$, secondary $\mathrm{RN}$, and target node. The target node is the device the position of which is to be estimated. A primary $\mathrm{RN}$ is an $\mathrm{RN}$ that performs TW-ToA ranging with the target node. On the other hand, a secondary RN listens to the signals transmitted both from the target and the primary reference nodes during TWToA ranging, and then calculates the difference between the arrival times of those signals. Overall, the position is estimated based on range information obtained from TW-ToA operations by the primary RNs and the TDoA information obtained from secondary RNs. It is important to note that the TDoA information used in the considered cooperative technique is quite different from the one in conventional TDoA systems described above. For conventional TDoA calculations, the RNs that are involved in TDoA calculation must be synchronized, whereas there is no such a requirement in the cooperative scheme since the TDoA is defined for one RN. It should also be noted that in the cooperative technique, secondary RNs do not need any additional transmissions from the main network; they just utilize the signals that are already being transmitted by the target node and the primary RNs during TW-ToA based ranging. In addition, the TW-ToA and TDoA measurements are correlated in the cooperative positioning protocol.

The cooperative positioning algorithm described above can be applied to both remote-positioning (tracking) and selfpositioning (navigation) systems [9]. For example, in a tracking scenario, the primary $\mathrm{RN}$ initiates the ranging protocol with the target node by transmitting a signal (Fig. 1). The target, upon reception of the signal, sends back a reply, thereby completing a TW-ToA signal exchange. A secondary RN that is capable of hearing both signals records the difference between their arrival times and passes the TDoA information to the primary RN. By this way, both ToA and TDoA information is obtained, which can be used in the position estimation of the target node as studied in Section III-B.

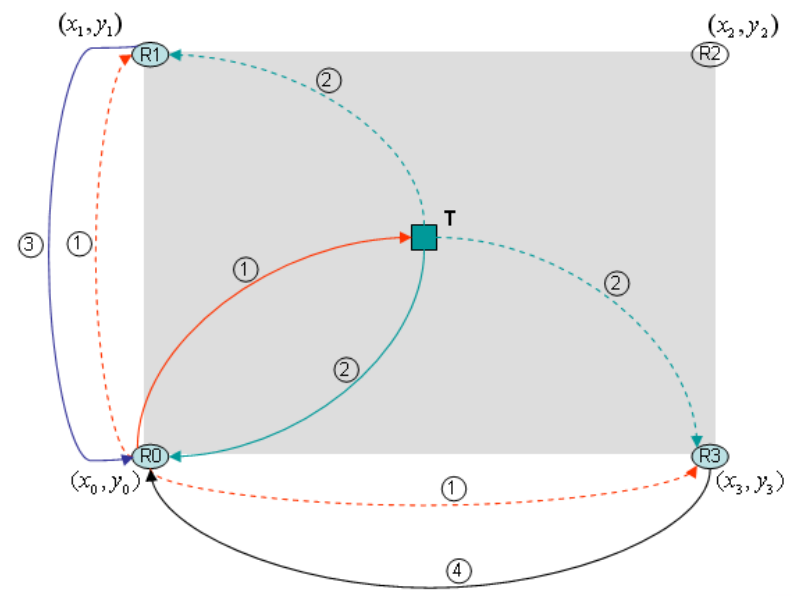

Fig. 1. Illustration of the cooperative positioning protocol. The primary RN $R O$ initiates the positioning protocol by sending a range request message. The target transmits a range reply. The secondary RNs $R 1$ and $R 3$ record the arrival times of the range request and reply messages, and report them back to $R O$. 


\section{A. Measurements Model}

First, consider a conventional TW-ToA estimation protocol, in which $N$ primary RNs are exchanging messages with the target node. As discussed in Section II, each ToA estimate obtained by this protocol includes two noise terms related to ToA estimations at the related two nodes. Therefore, the ToA estimates (measurements) can be expressed as

$$
z_{i}=r_{i}+\frac{n_{T, i}}{2}-\frac{n_{i, T}}{2},
$$

for $i=1, \ldots, N$, where $r_{i}=\sqrt{\left(x_{i}-x_{T}\right)^{2}+\left(y_{i}-y_{T}\right)^{2}}$ is the distance between the $i$ th primary $\mathrm{RN}$ and the target, $n_{i, T}$ is the error due to ToA estimation at the target node for the signal transmitted from the $i$ th primary $\mathrm{RN}$, and $n_{T, i}$ is the error due to ToA estimation at the $i$ th primary $\mathrm{RN}$ for the signal transmitted from the target node.

For the cooperative positioning technique, consider a scenario in which there are $N$ primary RNs and $M$ secondary RNs. The primary RNs are implementing the TW-ToA protocol with the target node; hence, they provide $N$ measurements as in (1). In addition, some secondary RNs can hear the signals that are employed in the TW-ToA protocols and provide additional position information. For each primary $\mathrm{RN}$ that initiates a TW-ToA protocol with the target node, $M$ additional measurements are obtained by the secondary RNs. Specifically, when the $i$ th primary $\mathrm{RN}$ is performing a TWToA protocol with the target node, the $j$ th secondary $\mathrm{RN}$ first hears the signal sent by the $i$ th primary RN and estimates its arrival time according to its local clock. Note that this signal arrives at the $j$ th secondary $\mathrm{RN}$ after $r_{i j} / c$ seconds, where $r_{i j}$ is the distance between the $i$ th primary $\mathrm{RN}$ and the $j$ th secondary $\mathrm{RN}$, and $c$ is the speed of light. When the signal arrives at the target node (after $r_{i} / c$ seconds), the target node prepares a reply message and sends it back to the $i$ th primary RN. The $j$ th secondary RN also listens to this signal, and estimates its arrival time. Note that this signal arrives at the secondary $\mathrm{RN}$ after $r_{i} / c+T_{t a}+\tilde{r}_{j} / c$ seconds, where $T_{t a}$ is the turn-around time and $\tilde{r}_{j}$ is the distance between the target and the $j$ th secondary RN. Then, the $j$ th secondary RN calculates the difference between the arrival times of the signals it receives from the target and the $i$ th primary $\mathrm{RN}$, and sends this information to the $i$ th primary $\mathrm{RN}$. This time difference information can be modeled as ${ }^{1}$

$$
z_{i}^{j}=r_{i}+\tilde{r}_{j}+\tilde{n}_{i, T, j}-n_{i, T}-\tilde{n}_{i, j},
$$

for $j=1, \ldots, M$, where $n_{i, T}$ is as in (1), and $\tilde{n}_{i, T, j}$ and $\tilde{n}_{i, j}$ represent the errors due to ToA estimation at the $j$ th secondary $\mathrm{RN}$ for the signals transmitted from the target node and from the $i$ th primary $\mathrm{RN}$, respectively. Note that $n_{i, j}$ and $n_{l, k}\left(\tilde{n}_{i, j}\right.$ and $\left.\tilde{n}_{l, k}\right)$ are independent for $(i, j) \neq(l, k)$, and $n_{i, j}$ and $\tilde{n}_{l, k}$ are independent for $\forall i, j, l, k$. In multipath channels, typically the first arriving path refers to the direct distance. However, in some channels the first arriving path may not be the strongest. Therefore, a search-back for the leading path is needed after acquisition [19]. Depending on the signal-to-noise ratio (SNR) and available processing gain, some timing error is induced in

\footnotetext{
${ }^{1}$ Since the positions of the RNs are known, $r_{i j}$ is a known value; hence, it is not included in (2) for convenience. In addition, the turn-around time is compensated at the $i$ th primary $\mathrm{RN}$ as it gets the turn-around time information from the target node.
}

the result of the search-back. The noise parameters in (1) and (2) are used to model such errors. From (1) and (2), the vector of observations for the cooperative positioning algorithm can be expressed as

$\mathbf{z}=\left[\begin{array}{lllllllllllllll}z_{1} & z_{2} & \cdots & z_{N} & z_{1}^{1} & \cdots & z_{1}^{M} & z_{2}^{1} & \cdots & z_{2}^{M} & \cdots & z_{N}^{1} & \cdots & z_{N}^{M}\end{array}\right]^{T}$.

\section{B. Position Estimation}

In this section, the maximum likelihood estimator (MLE) for the target position is derived. To that aim, the probability density function (PDF) of $\mathbf{z}$ in (3) should be obtained first. Note that the components of $\mathbf{z}$ are not independent due to the presence of the noise terms at the target node (i.e., $\left.n_{i, T}\right)$. However, conditioned on those noise components; that is, given $\mathbf{n}_{T}=\left[n_{1, T} \cdots n_{N, T}\right]$, the components of $\mathbf{z}$ are independent; hence, the PDF of $\mathbf{z}$ can be calculated easily based on the following expression:

$$
p_{\boldsymbol{\theta}}(\mathbf{z})=\int p_{\boldsymbol{\theta}}\left(\mathbf{z} \mid \mathbf{n}_{T}\right) f_{\boldsymbol{\theta}}\left(\mathbf{n}_{T}\right) d \mathbf{n}_{T},
$$

where $f_{\boldsymbol{\theta}}\left(\mathbf{n}_{T}\right)$ is the PDF of the noise components due to the measurements at the target node. After some manipulation, the PDF can be obtained and the MLE for the target position is expressed as (please see Appendix A)

$$
\begin{gathered}
\arg \min _{[x y]} \sum_{i=1}^{N}\left\{\left(\frac{2}{\sigma_{i}^{2}}-\frac{1}{a_{i} \sigma_{i}^{4}}\right)\left(z_{i}-r_{i}\right)^{2}-\frac{1}{a_{i}}\left(\sum_{j=1}^{M} \frac{\left(z_{i}^{j}-r_{i}-\tilde{r}_{j}\right)}{4 \tilde{\sigma}_{j}^{2}}\right)^{2}\right. \\
\left.-\frac{\left(z_{i}-r_{i}\right)}{a_{i} \sigma_{i}^{2}} \sum_{j=1}^{M} \frac{\left(z_{i}^{j}-r_{i}-\tilde{r}_{j}\right)}{2 \tilde{\sigma}_{j}^{2}}+\sum_{j=1}^{M} \frac{\left(z_{i}^{j}-r_{i}-\tilde{r}_{j}\right)^{2}}{4 \tilde{\sigma}_{j}^{2}}\right\}
\end{gathered}
$$

where $a_{i} \doteq \frac{1}{2 \sigma_{T}^{2}}+\frac{1}{2 \sigma_{i}^{2}}+\sum_{j=1}^{M} \frac{1}{4 \tilde{\sigma}_{j}^{2}}$.

Note that the result in (5) can be considered as an extension of the nonlinear least-squares (NLS) estimator for the cases of independent and identically distributed measurements [9]. In addition, as $\tilde{\sigma}_{j} \rightarrow \infty$, the MLE in (5) reduces to $\arg \min _{[x y]} \sum_{i=1}^{N} \frac{\left(z_{i}-r_{i}\right)^{2}}{\sigma_{i}^{2}+\sigma_{T}^{2}}$, which is the conventional NLS estimator for the TW-ToA based positioning algorithm [9].

\section{Performance Limits}

Theoretical lower bounds on position estimation are studied in terms of the CRLB. The derivation of a closed-form CRLB expression is useful also for network planning, which can optimize placement of primary and secondary RNs to meet certain accuracy requirements. The CRLB is calculated from inverse of the Fisher information matrix, which is derived from the conditional distribution of the observation vector $\mathbf{z}$ given the target coordinates vector $\boldsymbol{\theta}=\left[x_{T} y_{T}\right]$ [20]. The conditional distribution of $\mathbf{z}$ given $\boldsymbol{\theta}$ can be obtained from (1)-(3) as

$$
\mathbf{z} \mid \boldsymbol{\theta} \sim \mathcal{N}(\boldsymbol{\mu}(\boldsymbol{\theta}), \mathbf{C}),
$$

where $\boldsymbol{\mu}(\boldsymbol{\theta})$ is the mean of the observation vector, and $\mathbf{C} \in$ $\mathcal{S}_{(N+1) M}$ represents the covariance matrix ${ }^{5}$. From (1)-(3), the mean vector can be obtained as

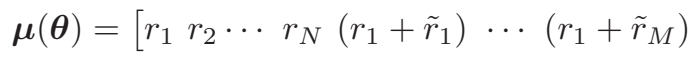

$$
\begin{aligned}
& \left.\cdots\left(r_{N}+\tilde{r}_{1}\right) \cdots\left(r_{N}+\tilde{r}_{M}\right)\right]^{T},
\end{aligned}
$$

${ }^{5}$ The set of all $m$-by- $n$ real matrices are denoted by $\mathcal{S}_{n, m}$ for $m \neq n$, and by $\mathcal{S}_{n}$ for $n=m$. 
where $r_{i}=\sqrt{\left(x_{i}-x_{T}\right)^{2}+\left(y_{i}-y_{T}\right)^{2}}$ and $r_{j}=$ $\sqrt{\left(\tilde{x}_{j}-x_{T}\right)^{2}+\left(\tilde{y}_{j}-y_{T}\right)^{2}}$ with $\left[\tilde{x}_{j} \tilde{y}_{j}\right]$ denoting the position of the $j$ th secondary RN. Similarly, the elements of $\mathbf{C}$ can be computed from (1) and (2) as

$$
\begin{aligned}
\mathrm{E}\left\{\left(z_{i}-\mathrm{E}\left\{z_{i}\right\}\right)\left(z_{j}-\mathrm{E}\left\{z_{j}\right\}\right)\right\} & = \begin{cases}\frac{\sigma_{i}^{2}+\sigma_{T}^{2}}{4}, & i=j \\
0, & i \neq j\end{cases} \\
\mathrm{E}\left\{\left(z_{i}-\mathrm{E}\left\{z_{i}\right\}\right)\left(z_{j}^{k}-\mathrm{E}\left\{z_{j}^{k}\right\}\right)\right\} & = \begin{cases}\frac{\sigma_{T}^{2}}{2}, & i=j \\
0, & i \neq j\end{cases} \\
\mathrm{E}\left\{\left(z_{i}^{k}-\mathrm{E}\left\{z_{i}^{k}\right\}\right)\left(z_{j}^{m}-\mathrm{E}\left\{z_{j}^{m}\right\}\right)\right\} & = \begin{cases}\sigma_{T}^{2}+2 \tilde{\sigma}_{k}^{2}, & i=j, k=m \\
\sigma_{T}^{2}, & i=j, k \neq m \\
0, & i \neq j\end{cases}
\end{aligned}
$$

Let $\mathbf{C}$ be partitioned as $\mathbf{C}=\left[\begin{array}{l|l}\mathbf{C}_{11} & \mathbf{C}_{12} \\ \hline \mathbf{C}_{21} & \mathbf{C}_{22}\end{array}\right]$ with $\mathbf{C}_{11} \in$ $\mathcal{S}_{N}, \mathbf{C}_{12}=\mathbf{C}_{21}^{T} \in \mathcal{S}_{N, M N}$, and $\mathbf{C}_{22} \in \mathcal{S}_{N M}$. These submatrices can be obtained from (8)-(10) as

$$
\begin{gathered}
\mathbf{C}_{11}=\operatorname{diag}\left\{\frac{\sigma_{1}^{2}+\sigma_{T}^{2}}{4}, \ldots, \frac{\sigma_{N}^{2}+\sigma_{T}^{2}}{4}\right\} \\
\mathbf{C}_{12}=\frac{\sigma_{T}^{2}}{2}\left[\begin{array}{ccccccccccccc}
1 & 1 & \cdots & 1 & 0 & 0 & \cdots & 0 & \cdots & 0 & 0 & \cdots & 0 \\
0 & 0 & \cdots & 0 & 1 & 1 & \cdots & 1 & \cdots & 0 & 0 & \cdots & 0 \\
\vdots & & \vdots & & \vdots & \vdots \\
0 & 0 & \cdots & 0 & 0 & 0 & \cdots & 0 & \cdots & 1 & 1 & \cdots & \\
& & & \cdots
\end{array}\right]
\end{gathered}
$$

$$
\mathbf{C}_{22}=\left[\begin{array}{cccc}
\mathbf{B} & \mathbf{Z} & \cdots & \mathbf{Z} \\
\mathbf{Z} & \mathbf{B} & \cdots & \mathbf{Z} \\
\vdots & \vdots & \vdots & \vdots \\
\mathbf{Z} & \mathbf{Z} & \cdots & \mathbf{B}
\end{array}\right]
$$

where $\mathbf{B}, \mathbf{Z} \in \mathcal{S}_{M}, \mathbf{Z}=\mathbf{0}_{M \times M}$, and

$$
\mathbf{B}=\sigma_{T}^{2} \mathbf{1}_{M \times M}+2 \operatorname{diag}\left\{\tilde{\sigma}_{1}^{2}, \ldots, \tilde{\sigma}_{M}^{2}\right\} .
$$

For the statistical model in (6), the elements of the Fisher information matrix can be calculated from [21]

$$
[I(\boldsymbol{\theta})]_{m n}=\left[\frac{\partial \boldsymbol{\mu}(\boldsymbol{\theta})}{\partial \theta_{m}}\right]^{T} \mathbf{C}^{-1}\left[\frac{\partial \boldsymbol{\mu}(\boldsymbol{\theta})}{\partial \theta_{n}}\right],
$$

for $m, n=1,2$, or equivalently

$$
[I(\boldsymbol{\theta})]_{m n} \sum_{j=1}^{L} \sum_{i=1}^{L} h_{i j} \frac{\partial[\boldsymbol{\mu}(\boldsymbol{\theta})]_{i}}{\partial \theta_{m}} \frac{\partial[\boldsymbol{\mu}(\boldsymbol{\theta})]_{j}}{\partial \theta_{n}},
$$

where $h_{i j}=\left[\mathbf{C}^{-1}\right]_{i j}$ and $L=N(M+1)$.

From (7), an explicit expression of the elements of $\partial \boldsymbol{\mu}(\boldsymbol{\theta}) / \partial \theta_{m}$ can be obtained after some manipulation as (note that $\theta_{1} \doteq x_{T}$ and $\left.\theta_{2} \doteq y_{T}\right)$

$$
\begin{aligned}
& {\left[\frac{\partial \boldsymbol{\mu}(\boldsymbol{\theta})}{\partial x_{T}}\right]_{i} \begin{cases}\left(x_{T}-x_{i}\right) / r_{i}, & i=1, \ldots, N \\
\frac{x_{T}-x_{f(i)}}{r_{f(i)}}+\frac{x_{T}-\tilde{x}_{g(i)}}{\tilde{r}_{g(i)}}, & i=N+1, \ldots, N(M+1)\end{cases} } \\
& {\left[\frac{\partial \boldsymbol{\mu}(\boldsymbol{\theta})}{\partial y_{T}}\right]_{i} \begin{cases}\left(y_{T}-y_{i}\right) / r_{i}, & i=1, \ldots, N \\
\frac{y_{T}-y_{f(i)}}{r_{f(i)}}+\frac{y_{T}-\tilde{y}_{g(i)}}{\tilde{r}_{g(i)}}, & i=N+1, \ldots, N(M+1)\end{cases} }
\end{aligned}
$$

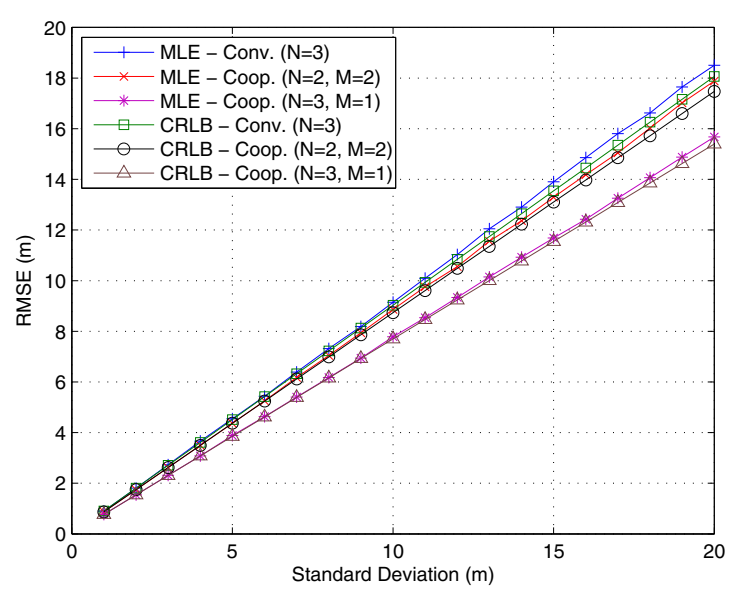

Fig. 2. The MLEs and CRLBs for the cooperative and the conventional algorithms.

where $f(i)=\lceil(i-N) / M\rceil$ and $g(i)=(i-N-1) \bmod M+1{ }^{6}$ Then, the CRLB on the MSE of an unbiased position estimator $\hat{\boldsymbol{\theta}}$ is given by

$$
\mathrm{E}\left\{\|\hat{\boldsymbol{\theta}}-\boldsymbol{\theta}\|^{2}\right\} \geq \frac{[I(\boldsymbol{\theta})]_{11}+[I(\boldsymbol{\theta})]_{22}}{[I(\boldsymbol{\theta})]_{11}[I(\boldsymbol{\theta})]_{22}-[I(\boldsymbol{\theta})]_{12}^{2}} .
$$

In the conventional TW-ToA based positioning scheme with $N$ reference nodes, the elements of the $N \times N$ covariance matrix $\mathbf{C}_{t w}$ are given by

$$
\left[\mathbf{C}_{\mathrm{tw}}\right]_{i j}=\left\{\begin{array}{ll}
\sigma_{\gamma_{i}}^{2}, & i=j \\
0, & i \neq j
\end{array},\right.
$$

where $\sigma_{\gamma_{i}}^{2}=\left(\sigma_{i}^{2}+\sigma_{T}^{2}\right) / 4$. Then, the elements of the FIM are given by

$$
\begin{aligned}
& {[I(\boldsymbol{\theta})]_{11}=\sum_{i=1}^{N} \frac{1}{\sigma_{\gamma_{i}}^{2}} \frac{\left(x-x_{i}\right)^{2}}{r_{i}^{2}},} \\
& {[I(\boldsymbol{\theta})]_{22}=\sum_{i=1}^{N} \frac{1}{\sigma_{\gamma_{i}}^{2}} \frac{\left(y-y_{i}\right)^{2}}{r_{i}^{2}},} \\
& {[I(\boldsymbol{\theta})]_{12}=\sum_{i=1}^{N} \frac{1}{\sigma_{\gamma_{i}}^{2}} \frac{\left(y-y_{i}\right)\left(x-x_{i}\right)}{r_{i}^{2}} .}
\end{aligned}
$$

\section{Simulation Results}

For the simulations, four reference nodes (RNs) are placed at the corners of a $100 \mathrm{~m} \times 100 \mathrm{~m}$ square, and they are labeled as $\mathrm{RN}-1, \mathrm{RN}-2, \mathrm{RN}-3$ and $\mathrm{RN}-4$, which are at positions $(50,-50)$, $(50,50),(-50,50)$ and $(-50,-50)$, respectively (all in meters). The target nodes are located inside the square over a grid of $19 \times 19$ (i.e., 5-meter spacing in each direction). For each analysis, the average error values are obtained by taking an average over various target positions and 100 independent noise realizations for each target position. In addition, the noise variances at different nodes are taken to be the same.

\footnotetext{
${ }^{6}\lceil x\rceil$ represents the smallest integer that is larger than or equal to $x$, and $x \bmod y$ denotes the remainder of the division of $x$ by $y$.
} 


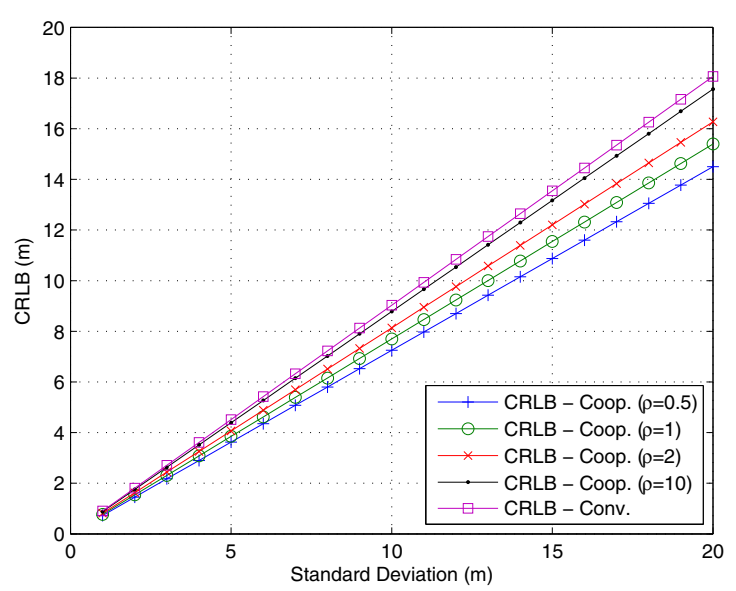

Fig. 3. CRLB vs. standard deviation for the cooperative and the conventional algorithms for various noise levels at the secondary $\mathrm{RN}(N=3, M=1)$.

In Fig. 2, the performance of the MLEs and the CRLBs are illustrated for both the conventional TW-ToA based and the cooperative positioning algorithms. For the TW-ToA, RN$1, \mathrm{RN}-2$ and $\mathrm{RN}-3$ are used to locate the targets $(N=3)$, whereas two different cases are considered for the cooperative algorithm. In the first case, RN-1, RN-2 and RN-3 are used as the primary RNs and RN-4 acts as a secondary $\mathrm{RN}(N=3$, $M=1)$. In the second case, $\mathrm{RN}-1$ and $\mathrm{RN}-3$ are used as the primary RNs and $\mathrm{RN}-2$ and $\mathrm{RN}-4$ are used as the

Fig. 2 show that the accuracy can be increased significantly with the cooperation of RN-4 (about 2 meters for large noise variances). The additional message overload to the system (compared to the conventional algorithm) is just 3 messages in that case. In the second case $(N=2, M=2)$, the target node can obtain position information by sending only 2 messages (considering a self-positioning scenario), which increases the battery life of the device. From Fig. 2, it is clear that the performance of the cooperative positioning algorithm for $N=2$ and $M=2$ is still better than the conventional one, despite using only 2 transmissions (3 transmissions are required in the conventional one). In addition, for all cases, it is observed that the MLEs get quite close to the CRLBs especially for small noise variances [20].

Next, the effects of the reliability of the measurements provided by the secondary RNs are quantified for the cooperative positioning. The scenario considered for Fig. 2 is assumed with $N=3$ and $M=1$, and the standard deviation of the measurement noise related to the secondary RN (i.e., RN-4) is assumed to be $\rho$ times that of the measurement noise at the target node or at the primary RNs. Fig. 3 plots the CLRBs against the standard deviation of the noise at the target (or the primary RNs) for various values of $\rho$. Note that as $\rho$ increases, the measurements related to the secondary RN becomes more noisy, and the errors increase. For very large $\rho$ values, the secondary RN cannot provide any useful position information; hence, the performance of the cooperative algorithm converges to that of the conventional ("non-cooperative") one. In order to observe the effects of $\rho$ more explicitly, Fig. 4 plots the CRLBs against $\rho$ for two different noise standard deviations values related to the measurements at the target and the primary

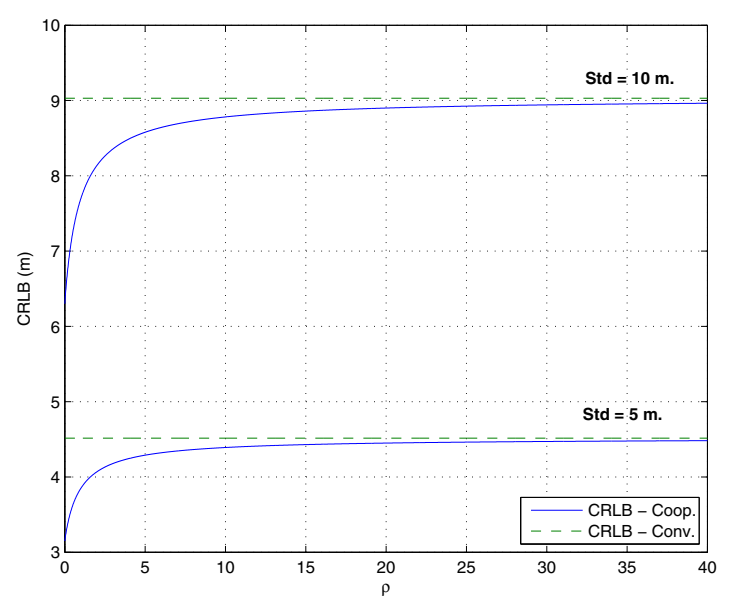

Fig. 4. The CRLB versus the noise coefficient $\rho$ for the measurement noise at the secondary $\mathrm{RN}(N=3, M=1)$.

RNs. It is again observed that as the amount of noise in the measurements related to the secondary $\mathrm{RN}$ increases, the performance of the cooperative algorithm falls down to that of the conventional one.

Finally, the cooperative and the conventional algorithms are compared in terms of positioning accuracy and communications cost for various scenarios. An environment is considered in the presence of 6 RNs located at $(50,-50)$, $(50,50),(-50,50),(-50,-50),(-50,0)$ and $(50,0)$ (all in meters). In Table I, the RMSEs of the MLEs, as well as the CRLBs, are calculated for the two algorithms in various scenarios, assuming a noise standard deviation $10 \mathrm{~m}$. for all the measurements. In addition, the total number of packets that are employed in the position estimation protocol are listed. Each scenario corresponds to $N$ primary RNs and $M$ secondary RNs, where the first $N$ of the positions $(50,-50)$, $(50,50),(-50,50),(-50,-50),(-50,0)$ and $(50,0)$ are selected as the primary $\mathrm{RN}$ positions and the next $M$ of them are selected as the secondary $\mathrm{RN}$ positions. Table I reveals various advantages of the cooperative approach. First, the cooperative approach provides position estimates even in the cases of 1 or 2 primary RNs by cooperating with the secondary RNs, whereas the conventional TW-ToA protocol needs at least 3 primary RNs. Second, positioning accuracy can be increased via the cooperative protocol at the expense of using more packets by cooperating with secondary RNs. As an example, for $N=4$, the RMSE of the MLE for the TW-ToA algorithm, which is equal to $7.28 \mathrm{~m}$., can be reduced to $6.10 \mathrm{~m}$. by cooperating with 2 secondary $\mathrm{RNs}(M=2)$. Third, in self-positioning (navigation) scenarios, the number of packets transmitted by the target node can be the important factor in determining the power consumption at the target node. In both algorithms, $N$ represents the number of packets that are transmitted by the target node. Hence, the cooperative algorithm can achieve similar (or, better) performance than the conventional one by consuming less power (i.e., using smaller number $N$ of primary RNs). For example, the RMSE of the MLE for the TW-ToA algorithm is equal to $9.03 \mathrm{~m}$. for $N=3$, whereas it is equal to $8.79 \mathrm{~m}$. for the cooperative algorithm 
TABLE I

COMPARISON OF COOPERATIVE AND CONVENTIONAL RANGING PROTOCOLS FOR VARIOUS NODE CONFIGURATIONS.

\begin{tabular}{|c|ccc|}
\hline & \multicolumn{3}{|c|}{ Cooperative/TW-ToA } \\
\hline Scenario & MLE $(\mathrm{m})$. & CRLB $(\mathrm{m})$. & \# \\
\hline \hline$N=1, M=2$ & $17.3 /-$ & $16.8 /-$ & $4 /-$ \\
\hline$N=1, M=3$ & $13.0 /-$ & $12.5 /-$ & $5 /-$ \\
\hline$N=1, M=4$ & $12.0 /-$ & $11.5 /-$ & $6 /-$ \\
\hline$N=1, M=5$ & $11.1 /-$ & $10.7 /-$ & $7 /-$ \\
\hline$N=2, M=1$ & $11.6 /-$ & $11.4 /-$ & $6 /-$ \\
\hline$N=2, M=2$ & $8.79 /-$ & $8.74 /-$ & $8 /-$ \\
\hline$N=2, M=3$ & $8.16 /-$ & $8.12 /-$ & $10 /-$ \\
\hline$N=2, M=4$ & $7.61 /-$ & $7.53 /-$ & $12 /-$ \\
\hline$N=3, M=1$ & $7.78 / 9.13$ & $7.70 / 9.03$ & $9 / 6$ \\
\hline$N=3, M=2$ & $7.28 / 9.13$ & $7.22 / 9.03$ & $12 / 6$ \\
\hline$N=3, M=3$ & $6.63 / 9.13$ & $6.55 / 9.03$ & $15 / 6$ \\
\hline$N=4, M=1$ & $6.71 / 7.28$ & $6.65 / 7.25$ & $12 / 8$ \\
\hline$N=4, M=2$ & $6.10 / 7.28$ & $6.04 / 7.25$ & $16 / 8$ \\
\hline$N=5, M=1$ & $5.95 / 6.59$ & $5.93 / 6.55$ & $15 / 10$ \\
\hline$N=6$ & $5.96 / 12$ & $5.93 / 5.93$ & $12 / 12$ \\
\hline
\end{tabular}

for $N=M=2$.

\section{CONCLuding Remarks}

The cooperative positioning scheme introduced in [1] has been investigated, which can provide improved accuracy by utilizing passive routers (secondary RNs) and offer position estimates with fewer numbers of transmissions than the conventional TW-ToA. Both the MLE and the CRLB have been derived in order to analyze the performance of the cooperative scheme, and simulation results have been presented to illustrate the improvements provided by the cooperative technique.

\section{APPENDIX}

\section{A. Derivation of the MLE in (5)}

In order to calculate the MLE based on (4), the expression for $p_{\boldsymbol{\theta}}(\mathbf{z})$ will be obtained first.

It is observed from (1) and (2) that given $\mathbf{n}_{T}=$ $\left[n_{1, T} \cdots n_{N, T}\right]$, the components of $\mathbf{z}$ are independent. Hence,

$$
\begin{aligned}
& p_{\boldsymbol{\theta}}\left(\mathbf{z} \mid \mathbf{n}_{T}\right)=\prod_{i=1}^{N} \frac{2}{\sqrt{2 \pi} \sigma_{i}} \exp \left\{-\frac{2}{\sigma_{i}^{2}}\left(z_{i}-r_{i}+\frac{n_{i, T}}{2}\right)^{2}\right\} \\
& \times \prod_{i=1}^{N} \prod_{j=1}^{M} \frac{1}{2 \sqrt{\pi} \tilde{\sigma}_{j}} \exp \left\{-\frac{1}{4 \tilde{\sigma}_{j}^{2}}\left(z_{i}^{j}-r_{i}+\tilde{r}_{j}+n_{i, T}\right)^{2}\right\} .
\end{aligned}
$$

The noise samples due to the target node, $\mathbf{n}_{T}=$ $\left[n_{1, T} \cdots n_{N, T}\right]$, are independent and identically distributed zero mean Gaussian variables with variance $\sigma_{T}^{2}$. Therefore, the PDF in (4) can be calculated from (24) as

$$
\begin{aligned}
& p_{\boldsymbol{\theta}}(\mathbf{z})=k \prod_{i=1}^{N} \int_{-\infty}^{\infty} \exp \left\{-\frac{2}{\sigma_{i}^{2}}\left(z_{i}-r_{i}+\frac{n_{i, T}}{2}\right)^{2}\right. \\
& \left.-\sum_{j=1}^{M} \frac{1}{4 \tilde{\sigma}_{i}^{2}}\left(z_{i}^{j}-r_{i}-\tilde{r}_{j}+n_{i, T}\right)^{2}-\frac{n_{T, i}^{2}}{2 \sigma_{T}^{2}}\right\} d n_{i, T},
\end{aligned}
$$

where $k$ is a constant that is independent of the measurements and the target position.
After some manipulation and using that fact that $\int_{-\infty}^{\infty} e^{-a x^{2}-2 b x} d x=\sqrt{\frac{\pi}{a}} e^{b^{2} / a}$ for $a>0$, the value of the target position $\left[\begin{array}{ll}x & y\end{array}\right]$ that maximizes the PDF in (25) can be obtained as in (5).

\section{REFERENCES}

[1] R. Fujiwara, K. Mizugaki, T. Nakagawa, D. Maeda, and M. Miyazaki "TOA/TDOA hybrid relative positioning system using UWB-IR," IEEE Radio and Wireless Week, San Diego, CA, Jan. 2009, pp. 679-682.

[2] J. Caffery, Jr., Wireless Location in CDMA Cellular Radio Systems, Kluwer Academic Publishers, Boston, 2000.

[3] K. Muthukrishnan, M. E. M. Lijding, and P. J. M. Havinga, "Towards smart surroundings: Enabling techniques and technologies for localization," in Proc. Int. Workshop on Location- and Context-Awareness, Oberpfaffenhofen, Germany, May 2005, pp. 350-362.

[4] Z. Sahinoglu, S. Gezici, I. Guvenc, Ultra-wideband Positioning Systems: Theoretical Limits, Ranging Algorithms and Protocols, Cambridge University Press, New York, 2008.

[5] S. Gezici and H. V. Poor, "Position estimation via ultra-wideband signals," Proceedings of the IEEE (Special Issue on UWB Technology and Emerging Applications), vol. 97, no. 2, pp. 386-403, Feb. 2009

[6] D. Kelly and G. Shreve and D. Langford, "Fusing communications and positioning - Ultrawideband offers exciting possibilities," Time Domain Corporation, Aug. 1998.

[7] R. Fontana and S. J. Gunderson, "Ultra-wideband precision asset location system," In Proc. IEEE Conf. on Ultra-wideband Syst. and Technol. (UWBST), Baltimore, MD, May 2002, pp. 147-150.

[8] R. J. Fontana, "Experimental results from an ultrawideband precision geolocation system," Multispectral Solutions, Inc., May 2000, pp. 1-6.

[9] S. Gezici, "A survey on wireless position estimation," Wireless Personal Communications (Special Issue on Towards Global and Seamless Personal Navigation), vol. 44, no. 3, pp. 263-282, Feb. 2008.

[10] C. Fretzagias, M. Papadopouli, "Cooperative Location-sensing for Wireless Networks," In Proc. Second IEEE Annual Conf. on Pervasive Computing and Commun. (PERCOM), Orlando, FL, March 2004.

[11] Y. Shen, H. Wymeersch, and M. Z. Win, "Fundamental Limits of Wideband Cooperative Localization via Fisher Information," In Proc. IEEE Wireless Coomun. and Networking Conf. (WCNC), Hong Kong, March 2007, pp.3954-3958.

[12] C. L. F. Mayorga, F. D. Rosa, and S. A. Wardana, "Cooperative Positioning Techniques for Mobile Localization in 4G Cellular Networks," In Proc. IEEE Int. Conf. on Pervasive Services, Istanbul, Turkey, July 2007, pp.39-44.

[13] T. Hui, W. Shuang, and X. Huaiyao, "Localization using Cooperative AOA Approach," In Proc. IEEE Int. Conf. on Wireless Commun., Networking and Mobile Computing (WiCOM), Shanghai, China, Sept. 2007, pp.2416-2419.

[14] A. T. Ihler, J. W. Fisher, R. L. Moses, and A. S. Willsky, "Nonparametric Belief Propagation for Self-localization of Sensor Networks," IEEE Journal on Selected Areas in Communications, vol. 23, no. 4, pp. 809819, Apr. 2005.

[15] N. A. Alsindi, K. Pahlavan, B. Alavi, and X. Li, "A novel cooperative localization algorithm for indoor sensor networks," In Proc. 17th Annual IEEE Int. Symposium on Personal, Indoor and Mobile Radio Commun. (PIMRC), Helsinki, Finland, Sept. 2006, pp.1-6.

[16] IEEE P802.15.4a/D4 (Amendment of IEEE Std 802.15.4), "Part 15.4: Wireless Medium Access Control (MAC) and Physical Layer (PHY) Specifications for Low-Rate Wireless Personal Area Networks (LRWPANs)," July 2006.

[17] Z. Sahinoglu and S. Gezici, "Ranging in the IEEE 802.15.4a Standard," In Proc. IEEE Wireless and Microwave Tech. Conf. (WAMICON), Florida, pp. 1-5, May 2006.

[18] N. Patwari, J. N. Ash, S. Kyperountas, A. O. Hero III, R. L. Moses and N. S. Correal, "Locating the nodes: Cooperative localization in wireless sensor networks," IEEE Signal Processing Mag., vol. 22, no. 4, pp. 5469, July 2005

[19] I. Guvenc, S. Gezici, and Z. Sahinoglu, "Ultra-wideband range estimation: Theoretical limits and practical algorithms," In Proc. IEEE International Conference on Ultra-Wideband (ICUWB 2008), vol. 3, pp. 93-96, Hannover, Germany, Sep. 2008.

[20] H. V. Poor, An Introduction to Signal Detection and Estimation, Springer-Verlag, New York, 1994.

[21] S. M. Kay, Fundamentals of Statistical Signal Processing: Estimation Theory, Prentice Hall, New Jersey, 1998. 